\title{
Avaliação da atenção primária em Sapucaia do Sul: comparação entre o modelo tradicional e a Estratégia Saúde da Família
}

\section{Evaluation of primary care in Sapucaia do Sul: comparison between the traditional model and the Family Health Strategy}

\author{
Cassiane Prestes da Silveira Chazan ${ }^{1}$ (D) Juvenal Soares Dias da Costa' (D) \\ 'Universidade do Vale do Rio dos Sinos, Programa de Pós-graduação em Saúde Coletiva - São Leopoldo, RS, Brasil.
}

Como citar: Chazan CPS, Dias-da-Costa JS. Avaliação da atenção primária em Sapucaia do Sul: comparação entre o modelo tradicional e a Estratégia Saúde da Família. Cad Saúde Colet, 2021;29(1):98-109. https://doi.org/10.1590/1414-462X202129010362

\begin{abstract}
Resumo
Introdução: O uso de métodos de avalição é uma forma de qualificação do sistema de saúde. Objetivo: Avaliou-se a qualidade dos serviços de atenção primária à saúde e compararam-se os atributos entre as unidades básicas de saúde com Estratégia Saúde da Família (ESF) com aquelas de modelo tradicional, em Sapucaia do Sul, RS, permitindo a identificação dos atributos mais vulneráveis. Método: Trata-se de estudo transversal de avaliação da APS utilizando-se o instrumento PCATool - Brasil, versão profissionais de saúde. Os respondentes foram enfermeiros da rede. As unidades foram comparadas mediante o uso de médias dos escores observados nos atributos e seus intervalos de confiança de $95 \%$. Resultados: Das 24 unidades avaliadas, 20 (83,3\%) apresentaram escore geral médio maior que 6,60. O escore geral das unidades com ESF foi 7,24 (IC95\% 7,0-7,3), enquanto a média das BHU tradicionais foi 6,70 (IC95\% $6,1-7,0)$. Na maioria dos atributos, houve melhor desempenho nas equipes com ESF, embora a diferença tenha sido constatada nos atributos integralidade-serviços disponíveis e orientação comunitária. A acessibilidade atingiu o desempenho menos satisfatório nos dois modelos. Conclusão: Apontou-se a necessidade de se melhorar o acesso e de se incrementar o sistema de referência, e foram mostradas ações para aperfeiçoar o desempenho. Palavras-chave: atenção primária à saúde; avaliação em saúde; estratégia saúde da família.
\end{abstract}

\begin{abstract}
Introduction: The use of evaluation methods is a way of qualifying the health system. Objective: The objective of the present study was to evaluate the quality of primary health care (PHC) services and compare health units with Family Health Strategy with those units with a traditional model, in Sapucaia do Sul, RS. This study identified the most vulnerable attributes among units. Methods: This is a crosssectional study of PHC assessment using the PCATool - Brazil instrument, version of health professionals. Respondents were network nurses. The units were compared using the average scores observed in the attributes and their 95\% confidence intervals. Results: 20 (83.3\%) of the 24 units evaluated, had an average general score greater than 6.60. The general score of units with FHS was 7.24 (95\% Cl 7.0-7.3), while the average of traditional basic health units (BHU) was 6.70 (95\% Cl 6.1-7.0). In most of the attributes, there was a better performance in teams with FHS, although the difference was found in the integrality attributes - available services and community guidance. Accessibility achieved the least satisfactory performance in both models. Conclusion: The need was pointed out to improve access, to increase the reference system, and actions were shown to improve performance.
\end{abstract}

Keywords: primary health care; health evaluation; family health strategy.

Trabalho realizado no Programa de Pós-graduação em Saúde Coletiva da Universidade do Vale do Rio dos Sinos - São Leopoldo, RS, Brasil.

Correspondência: Juvenal Soares Dias da Costa. E-mail: episoares@terra.com.br

Fonte de financiamento: nenhuma.

Conflito de interesses: Cassiane Prestes da Silveira Chazan foi funcionária da Secretaria Municipal de Saúde de Sapucaia do Sul.

Recebido em: Set. 03, 2018. Aceito em: Fev. 29, 2020
Este é um artigo publicado em acesso aberto (Open Access) sob a licença Creative Commons Attribution, que permite uso, distribuição e reprodução em qualquer meio, sem restrições desde que o trabalho original seja corretamente citado. 


\section{INTRODUÇÃO}

Desde a implantação do Sistema Único constatou-se o aumento da utilização de serviços de saúde no Brasil, principalmente no nível da atenção primária'. Entretanto, desde a década de 1990, verificaram-se problemas relacionados à qualidade da atenção e resolutividade da rede de unidades básicas de saúde, marcadamente ampliada em sua oferta pelos municípios². Como tentativa de se modificar o modelo assistencial, aumentar a efetividade e o desempenho da atenção primária, o Ministério da Saúde organizou o Programa Saúde da Família que evoluiu para Estratégia Saúde da Família (ESF), com franca expansão em todo o país s.4. $^{3,4}$

O Ministério da Saúde tem sido o principal incentivador para a implantação de novas equipes de ESF, contudo sabe-se que o investimento necessário por parte dos municípios, para a manutenção da Estratégia, é superior quando comparado às unidades de saúde que atuam com o modelo tradicional, visto que, pelas legislações municipais, geralmente os valores agregados aos cargos e salários têm sido expressivamente mais elevados, bem como a quantidade de recursos humanos exigida ${ }^{5}$.

Esta realidade é encontrada em diversos municípios brasileiros, motivo pelo qual as discussões em relação à efetividade e o custo-benefício da ESF são pautas constantes nas comissões colegiadas.

No entanto, alguns estudos mostraram as vantagens da adoção da Estratégia Saúde da Família como forma de se qualificar o sistema de saúde. Por exemplo, com a implantação da ESF no Brasil, verificou-se a redução da mortalidade infantili ${ }^{6}$, aumento do número de consultas de pré-natal ${ }^{7}$ e a diminuição de internações por condições sensíveis à atenção primária ${ }^{8}$.

A melhora da qualidade dos serviços de saúde deve ser considerada como oportuna e pode ser acompanhada mediante a incorporação de estudos de avaliação permanente. Entretanto, estudos de avaliação sobre o desempenho da atenção primária à saúde (APS) ainda podem ser considerados incipientes no Brasil ${ }^{9,10}$.

Sabe-se que a avaliação dos sistemas e serviços de saúde permite à gestão identificar potencialidades e dificuldades, possibilitando o planejamento e ajustes, garantindo que a APS cumpra o papel de fazer a diferença na vida das pessoas ${ }^{11,12}$.

Diversos instrumentos de avaliação da atenção primária estão disponíveis, destacando-se o PCA-Tool, baseado no estabelecimento de escores para os atributos da atenção primária ${ }^{13}$.

Desde a década de 1990, o município de Sapucaia do Sul (RS) passou a expandir sua rede de saúde, priorizando o desenvolvimento da APS, atingindo cobertura populacional de $69,6 \%$ e totalizando investimento médio de $6 \%$ da arrecadação municipal nesse nível de atenção.

O objetivo do presente estudo foi avaliar a qualidade dos serviços de APS e comparar as unidades básicas de saúde com Estratégia Saúde da Família (ESF) com as unidades de saúde com modelo tradicional, no município de Sapucaia do Sul, por meio do PCATool - Brasil, versão profissionais de saúde. O estudo permitiu também a identificação dos atributos mais vulneráveis entre as unidades, como a possibilidade de sugerir ações para melhorar o desempenho da atenção primária.

\section{MÉTODO}

Foi realizado um estudo transversal para avaliação das unidades básicas de saúde do município de Sapucaia do Sul, Rio Grande do Sul, mediante a aplicação do instrumento PCATool - Brasil, versão profissionais de saúde.

O município está localizado na região metropolitana de Porto Alegre, situado a $20 \mathrm{~km}$ da capital e possui 138.357 habitantes. A cidade assumiu a gestão plena dos recursos vinculados à saúde em 2014 e, desde 2008, ampliou os investimentos visando à expansão e fortalecimento da APS.

A coleta de dados envolveu todos os 33 profissionais enfermeiros que compunham a rede de atenção primária do município. A escolha da categoria ocorreu devido à coordenação das unidades de saúde ficar a cargo do profissional enfermeiro. Parte da coleta dos dados ocorreu durante encontro promovido pela Secretaria Municipal de Saúde, em que foi apresentado o estudo e aplicado o instrumento. Os oito enfermeiros que não compareceram ao encontro 
foram contatados via telefone e a coleta aconteceu de forma presencial nas unidades de saúde onde atuavam.

Os dados foram coletados entre os meses de outubro de 2016 e janeiro de 2017. A rede de atenção primária à saúde do município, no momento da pesquisa, era constituída de 24 unidades básicas de saúde, totalizando 24 equipes de ESF (26 enfermeiros respondentes), visto que, em duas equipes de ESF, havia 2 enfermeiros atuando na mesma equipe e quatro unidades básicas de saúde atuando com o modelo tradicional (7 enfermeiros respondentes), sendo duas UBS com dois enfermeiros de referência e uma UBS com três enfermeiros responsáveis pela unidade.

O PCATool é um instrumento de avaliação da atenção primária nas versões: criança, adulto e profissionais. $\mathrm{O}$ instrumento foi baseado no modelo de avaliação da qualidade, na identificação e medida de aspectos ligados à estrutura, processo e resultado dos serviços de saúde ${ }^{14,15}$, sendo considerado um instrumento capaz de avaliar de forma fidedigna a APS ${ }^{16}$. O PCATool mensura a presença e a extensão de quatro atributos essenciais e de três atributos derivados da atenção primária definidos por Starfield ${ }^{17}$. Os quatro atributos considerados como essenciais são: a acessibilidade (acesso de primeiro contato), a longitudinalidade, a coordenação do cuidado e a integralidade. Os atributos derivados foram definidos como: orientação familiar, orientação comunitária e competência cultural. A aplicação do instrumento permitiu a construção de escores para cada atributo por meio da estruturação das respostas seguindo uma escala tipo Likert, possibilitando atribuir valores que variaram entre 1 "com certeza, não" e 4 "com certeza, sim".

O escore essencial foi obtido mediante o cálculo das médias dos atributos essenciais (acesso, longitudinalidade, coordenação do cuidado e integralidade) e o escore geral foi estabelecido utilizando a média destes escores incluindo os atributos derivados (orientação familiar e orientação comunitária). A escala Likert foi transformada em uma escala que variava entre 0 e 10 conforme orientações do instrumento original utilizando a seguinte fórmula:

$$
\frac{(\text { Escore }-1)}{(4-1)} \times 10
$$

Em consonância com as orientações do instrumento original, os escores gerais que apresentaram valores maiores ou iguais a 6,60 foram considerados como alto grau de orientação à APS. Nas UBS onde atuavam mais de um enfermeiro, os escores foram calculados por meio de média aritmética simples.

Os questionários foram digitados em Epidata, versão 3.1 e todas as análises, realizadas no programa SPSS, versão 23.0. As unidades com Estratégia Saúde da Família foram comparadas com as unidades com modelo tradicional mediante os intervalos de confiança a $95 \%$ das médias dos escores observados nos atributos. Foram calculados os intervalos de confiança a 95\% dos escores médios dos atributos, cujo erro padrão foi estabelecido mediante a fórmula: $D P /(\sqrt{ } n)$.

Foram descritos os itens que alcançaram pior desempenho, de forma que se pudessem identificar necessidades no sistema de saúde.

O projeto de pesquisa foi aprovado pelo Comitê de Ética e Pesquisa da Universidade do Vale do Rio dos Sinos (UNISINOS) com parecer número 1.745.251 e aprovado pelo colegiado gestor da Secretaria Municipal de Saúde de Sapucaia do Sul que expediu uma carta de anuência autorizando a pesquisa. Todos os profissionais participantes assinaram o Termo de Consentimento Livre e Esclarecido (TCLE) concordando com a participação na pesquisa.

\section{RESULTADOS}

Foram analisadas 24 unidades básicas de saúde e a média do escore geral foi de 7,15. Observou-se que a maior pontuação foi 8,26 e a menor foi 5,80 (Gráfico 1). Entre as unidades avaliadas, 20 (83,3\%) apresentaram escore geral médio maior que 6,60, sendo 17 unidades que atuavam com equipes de ESF e três unidades caracterizadas como modelo tradicional (Tabela 1).

A análise por domínios demonstrou que o atributo coordenação do cuidado (sistemas de informação) apresentou o maior escore, com média geral de 9,14, enquanto acessibilidade 


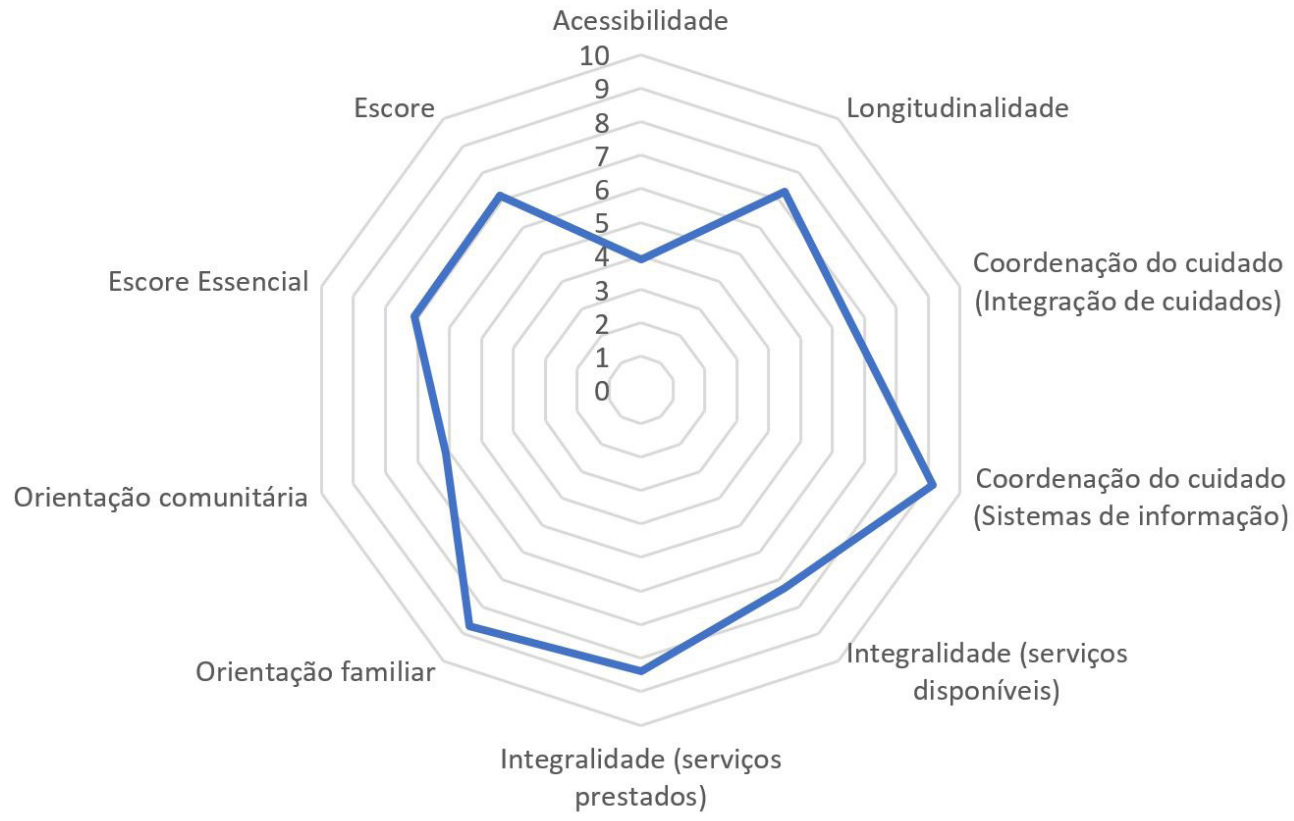

Gráfico 1. Média geral dos escores dos atributos mediante instrumento PCATool, Sapucaia do Sul, RS, 2016

alcançou o menor escore médio: 3,90. Outro atributo com baixo escore geral foi orientação comunitária, atingindo média de 6,12. O atributo coordenação do cuidado/integração do cuidado apresentou escore geral de 6,56, estabelecendo-se como limítrofe na orientação para a APS (Gráfico 1).

Foi realizada comparação entre as unidades que atuavam como saúde da família com as UBS de modelo tradicional. Observou-se que o escore geral das unidades com ESF foi 7,24 (IC95\% 7,0 a 7,3), enquanto a média das UBS tradicionais foi 6,70 (IC 95\% 6,1 a 7,0). Na comparação por componentes, verificou-se que quase todos os atributos atingiram maior média nas unidades que atuavam com ESF, com exceção de acessibilidade, atingindo 4,44 nas UBS tradicionais e 3,80 nas ESF. Os intervalos de confiança mostraram que os escores nos atributos integralidade (serviços disponíveis) e orientação comunitária foram mais elevados nos serviços com ESF. O atributo coordenação do cuidado/integração do cuidado obteve baixa pontuação, totalizando média de 6,52 em ambos os modelos de atenção (Tabela 2).

Em relação ao atributo acessibilidade, na análise dos nove itens que constituíam o componente, observou-se que os itens "A1" (seu serviço de saúde está aberto sábado ou domingo?) apresentaram média de resposta 1,03,"com certeza, não", mostrando que os serviços de saúde avaliados se encontravam em funcionamento somente em dias úteis. No item A5 (quando seu serviço de saúde está fechado existe um número de telefone para o qual os pacientes possam ligar quando adoecem?), dos 33 profissionais que participaram da pesquisa, 26 (78,0\%) apontaram como resposta a opção 1 "com certeza, não", alcançando média de resposta de 1,6. Outro item dentro do componente acessibilidade que apresentou baixo valor atribuído foi "A6" (quando seu serviço de saúde está fechado aos sábados e domingos e algum paciente fica doente, alguém do seu serviço o atende no mesmo dia?) a média das respostas também foi de 1,09 e o item "A7" (quando seu serviço de saúde está fechado à noite e algum paciente fica doente, alguém de seu serviço o atende naquela noite?) apresentou média de 1,1. Ainda em relação ao componente acessibilidade, no item A2 (seu serviço de saúde está aberto, pelo menos em alguns dias da semana até as 20 horas?), em apenas $6(25,0 \%)$ unidades de saúde, a resposta foi afirmativa "com certeza, sim", demonstrando assim que 18 (75,0\%) das unidades de saúde do município não estavam disponíveis à população após as 17 horas (Quadro 1). 


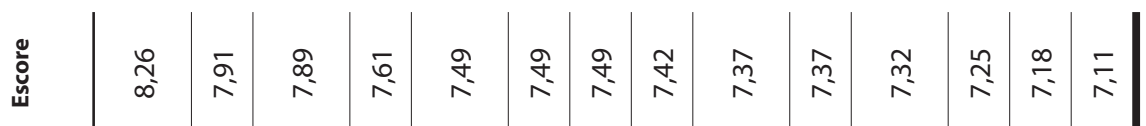

紊覀

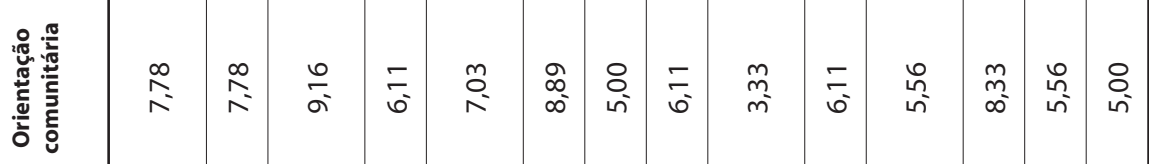

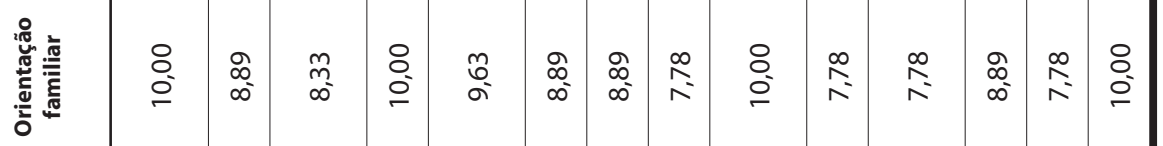

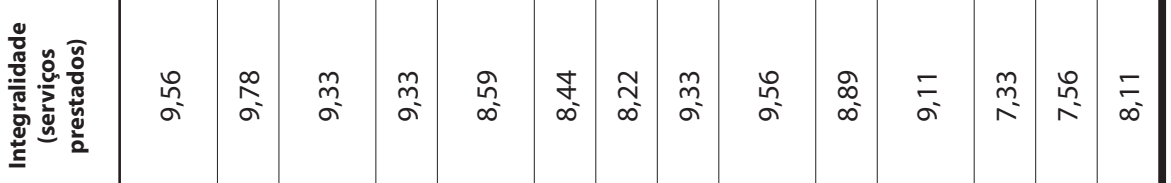

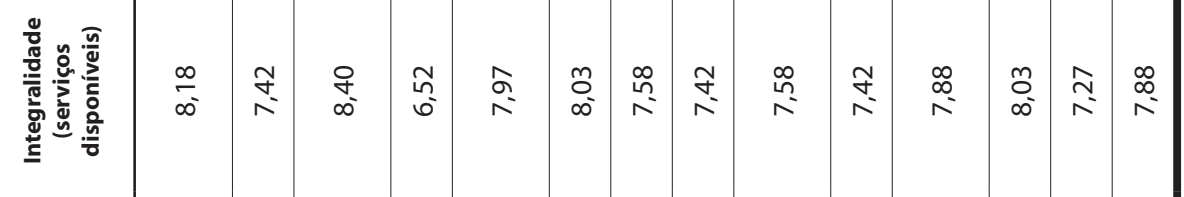

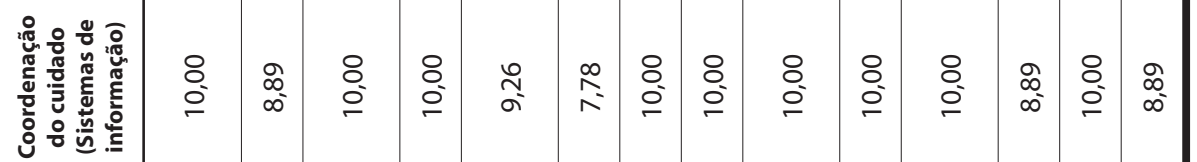

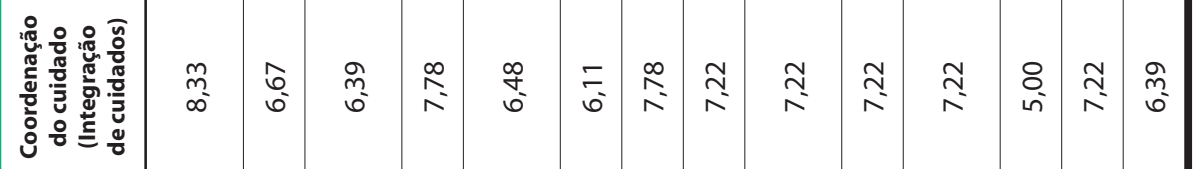

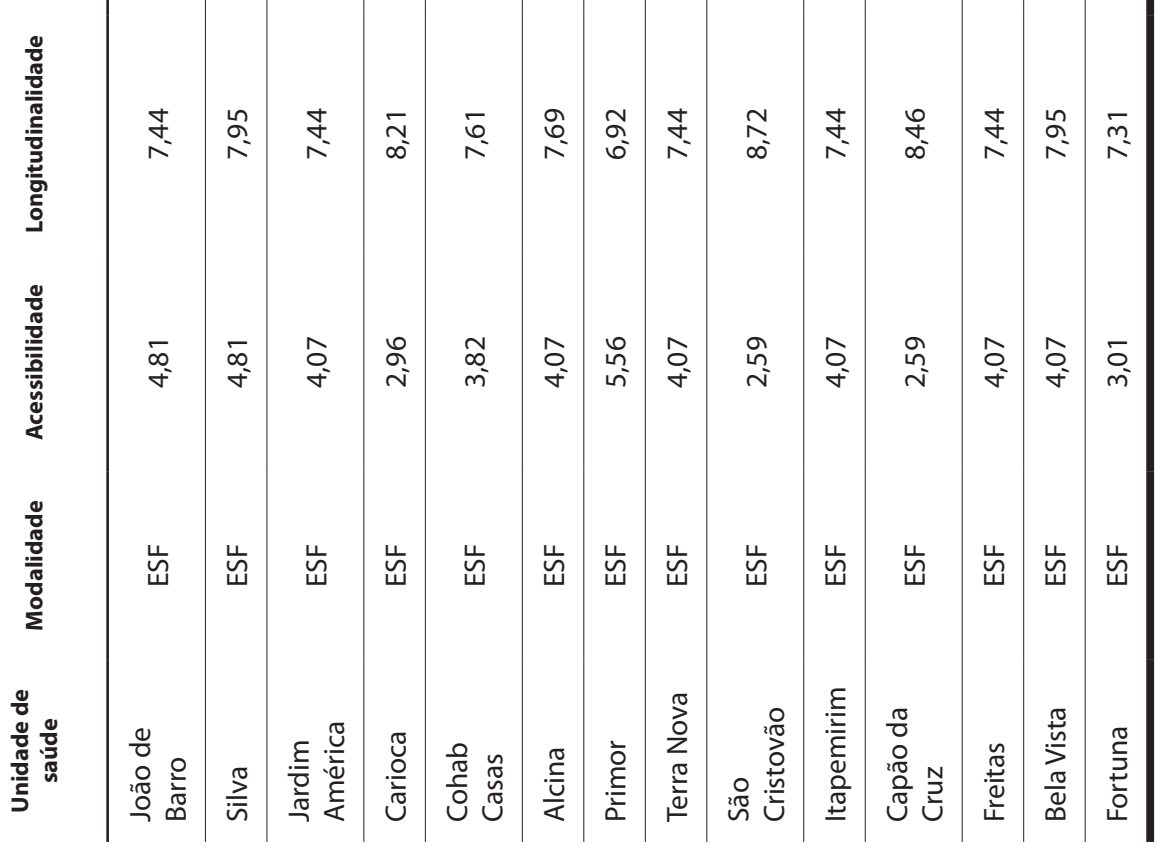




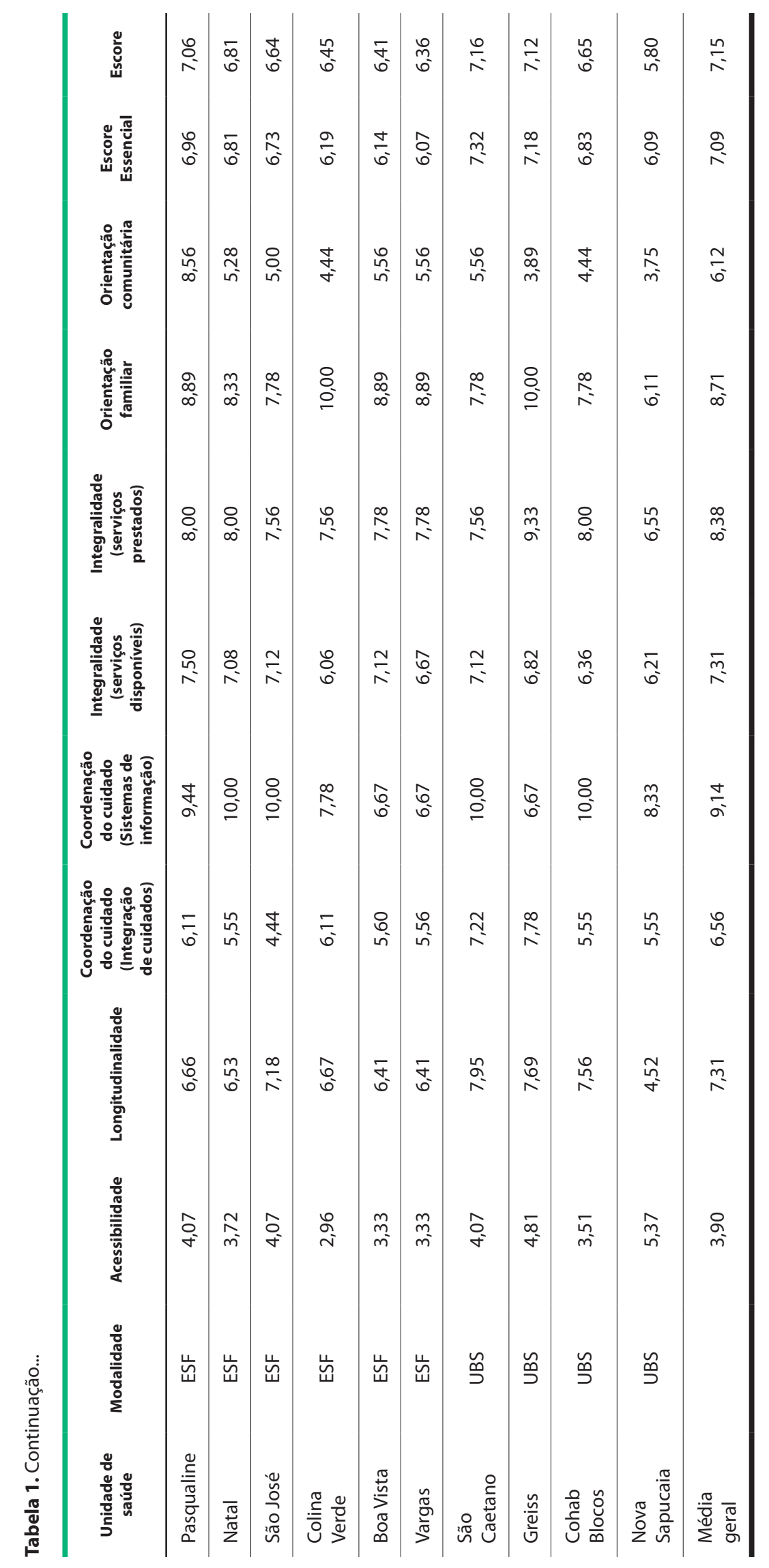


Tabela 2. Escore médio dos atributos com respectivos intervalos de confiança a 95\% de acordo com o tipo de serviço, Sapucaia do Sul, RS, 2016.

\begin{tabular}{lll}
\hline \multicolumn{1}{l}{ ESF(IC 95\%) } & USB Tradicional (IC95\%) \\
\hline Atributos Essenciais & & \\
\hline Acessibilidade & $3,80(3,5$ a 4,0$)$ & $4,44(3,6$ a 4,8) \\
\hline Longitudinalidade & $7,39(7,1$ a 7,6$)$ & $6,93(5,3$ a 7,8$)$ \\
\hline Coordenação do cuidado (Integração do cuidado) & $6,52(6,1$ a 6,7) & $6,52(5,4$ a 7,1$)$ \\
\hline Coordenação do cuidado (Sistemas de informação) & $9,21(8,7$ a 9,5) & $8,75(7,1$ a 9,5) \\
\hline Integralidade (Serviços Disponíveis) & $7,45(7,2$ a 7,6) & $6,62(6,1$ a 6,8) \\
\hline Integralidade (Serviços Prestados) & $8,49(8,1$ a 8,7) & $7,86(6,7$ a 8,5) \\
\hline Atributos Derivados & & \\
\hline Orientação Familiar & $8,87(8,5$ a 9,1) & $7,91(6,3$ a 8,7) \\
\hline Orientação Comunitária & $6,46(5,6$ a 6,9) & $4,41(3,6$ a 4,8) \\
\hline Escore Essencial & $7,14(6,8$ a 7,2) & $6,85(6,3$ a 7,1) \\
\hline Escore Geral & $7,24(7,0$ a 7,3) & $6,70(6,1$ a 7,0) \\
\hline
\end{tabular}

No atributo coordenação do cuidado/integração do cuidado, dos seis itens avaliados pelo instrumento, foi possível observar que o item " $\mathrm{C} 1$ " (você tem conhecimento de todas as consultas que seus pacientes fazem a especialistas ou serviços especializados?) apresentou média de resposta de 2,3 e "C5" (você recebe do especialista do serviço especializado informações úteis sobre o paciente encaminhado?) teve média de resposta de 1,6, apresentando assim as menores pontuações em relação aos demais itens, contribuindo para o baixo escore apresentado pelo componente (Quadro 1).

Ao se analisar os resultados obtidos para o componente integralidade - serviços disponíveis, o item E9 (sutura de um corte que necessita de pontos) obteve apenas 5 (15,0\%) respostas positivas "com certeza, sim", no E13 (colocação de tala), foram 2 (6,0\%) afirmações "com certeza, sim", no E14 (remoção de verrugas), 2 (6,0\%) respostas positivas"com certeza, sim" e no E18 (remoção de unhas encravadas), 5 (15,0\%) afirmações, apresentando assim escores abaixo do ponto de corte estabelecido pelo instrumento (Quadro 1).

Em relação ao componente integralidade - serviços prestados, no item F9 (pergunta se o paciente possui arma de fogo e orienta como guardá-la com segurança) apenas 2 (6,0\%) profissionais responderam "com certeza, sim" (Quadro 1).

$\mathrm{Na}$ análise do atributo orientação comunitária, foi possível observar que, no item $\mathrm{H} 4$ (faz pesquisas com os pacientes para ver se os serviços estão satisfazendo ou atendendo às necessidades das pessoas?), somente $3(9,1 \%)$, entre os profissionais respondentes, apontaram a resposta"com certeza, sim". Em relação ao item $\mathrm{H} 5$ (faz pesquisas na comunidade para identificar problemas de saúde que ela deveria conhecer), identificou-se que apenas $4(12,0 \%)$, dos 33 profissionais, responderam de forma totalmente afirmativa "com certeza, sim".

Em relação ao atributo longitudinalidade, quantidade expressiva de respondentes (36,3\%) afirmou a impossibilidade de os pacientes manterem contato por telefone com o médico ou enfermeiro que mais conhecessem. Esses respondentes admitiram ainda desconhecer todos os medicamentos que seus pacientes utilizavam.

\section{DISCUSSÃO}

Ao se analisar os resultados obtidos, foi possível observar que a maioria das UBS do município de Sapucaia do Sul apresentaram forte grau de orientação à APS, uma vez que 
Quadro 1. Descrição dos itens com menor pontuação entre os componentes/atributos, Sapucaia do Sul, RS, 2016.

\begin{tabular}{|c|c|}
\hline \multicolumn{2}{|c|}{ Componente/Atributo: Acesso de Primeiro Contato-Acessibilidade } \\
\hline Item & $\begin{array}{l}\text { Total de Respostas } \\
\text { positivas:"provavelmente } \\
\text { sim/com certeza, sim” (\%) }\end{array}$ \\
\hline A1: Seu serviço de saúde está aberto sábado ou domingo? & 0 \\
\hline $\begin{array}{l}\text { A2: Seu serviço de saúde está aberto, pelo menos em alguns dias da } \\
\text { semana até as } 20 \mathrm{~h} \text { ?) }\end{array}$ & $6(18,1 \%)$ \\
\hline $\begin{array}{l}\text { A5: Quando seu serviço de saúde está fechado existe um número de } \\
\text { telefone para qual os pacientes possam ligar quando adoecem? }\end{array}$ & $6(18,1 \%)$ \\
\hline $\begin{array}{l}\text { A6: Quando seu serviço de saúde está fechado aos sábados e domingos e } \\
\text { algum paciente fica doente, alguém do seu serviço o atende no mesmo dia? }\end{array}$ & 0 \\
\hline $\begin{array}{l}\text { A7: Quando seu serviço de saúde está fechado à noite e algum paciente fica } \\
\text { doente, alguém de seu serviço o atende naquela noite? }\end{array}$ & $1(3,0 \%)$ \\
\hline \multicolumn{2}{|l|}{ Componente/Atributo: coordenação do cuidado/integração do cuidado } \\
\hline $\begin{array}{l}\text { C1: Você tem conhecimento de todas as consultas que seus pacientes fazem } \\
\text { a especialistas ou serviços especializados? }\end{array}$ & $13(39,3 \%)$ \\
\hline $\begin{array}{l}\text { C5: Você recebe do especialista do serviço especializado informações úteis } \\
\text { sobre o paciente encaminhado? }\end{array}$ & $2(6,0 \%)$ \\
\hline \multicolumn{2}{|l|}{ Componente/Atributo: Integralidade - Serviços disponíveis } \\
\hline \multicolumn{2}{|l|}{$\begin{array}{l}\text { Item: Se um paciente tem necessidade de qualquer dos seguintes serviços } \\
\text { poderia obtê-lo no seu serviço de saúde? }\end{array}$} \\
\hline E9: sutura de um corte que necessita de pontos & $5(15,1 \%)$ \\
\hline E13: colocação de tala & $2(6,0 \%)$ \\
\hline E14: remoção de verrugas & $2(6,0 \%)$ \\
\hline E18: remoção de unhas encravadas & $5(15,1 \%)$ \\
\hline \multicolumn{2}{|l|}{ Componente/Atributo: Integralidade - Serviços prestados } \\
\hline $\begin{array}{l}\text { F9: Pergunta se o paciente possui arma de fogo e orienta como guardá-la } \\
\text { com segurança? }\end{array}$ & $2(6,0 \%)$ \\
\hline \multicolumn{2}{|l|}{ Componente/Atributo: Orientação comunitária } \\
\hline $\begin{array}{l}\text { H4: Faz pesquisas com os pacientes para ver se os serviços estão } \\
\text { satisfazendo (atendendo) as necessidades das pessoas? }\end{array}$ & $3(9,09 \%)$ \\
\hline $\begin{array}{l}\text { H5: Faz pesquisas na comunidade para identificar problemas de saúde que } \\
\text { ela deveria conhecer? }\end{array}$ & $4(12,1 \%)$ \\
\hline \multicolumn{2}{|l|}{ Componente/Atributo: Longitudinalidade } \\
\hline $\begin{array}{l}\text { B4: Se seus pacientes têm uma pergunta, podem telefonar e falar com o } \\
\text { médico ou enfermeiro que os conhece melhor? }\end{array}$ & $12(36,3 \%)$ \\
\hline B13: Você sabe todos os medicamentos que seus pacientes estão tomando? & $12(36,3 \%)$ \\
\hline
\end{tabular}

$20(83,3 \%)$ das 24 unidades avaliadas atingiram escore geral maior que 6,60, ponto de corte estabelecido pelo instrumento ${ }^{14,18}$.

Os intervalos de confiança não mostraram diferenças nas médias entre os escores gerais entre ESF e UBS tradicionais. Entretanto, deve-se ressaltar que os resultados apresentados 
revelaram um melhor desempenho das equipes que atuavam com o modelo de ESF em relação aos atributos da APS, com exceção do atributo acessibilidade, em que o escore foi maior entre as unidades do modelo tradicional, embora sem diferenças nos intervalos de confiança.

O escore com pontuação mais baixa foi acessibilidade. O componente foi estruturado para avaliar o acesso a partir de itens de difícil cumprimento por parte das equipes, como a abertura dos serviços apenas em dias úteis, a disponibilização de telefone e pagamento de profissional em horário não comercial e abertura das unidades após as 17 horas. Essas questões apresentam um desafio para a gestão municipal, uma vez que garantir a acessibilidade no primeiro contato pode melhorar os resultados em saúde e, principalmente, reduzir a procura por outros serviços de saúde como as urgências e os pronto-atendimentos, problema atualmente enfrentado na emergência do único hospital do município. Essa realidade também foi encontrada em diversos municípios brasileiros, em que o fortalecimento da APS se faz necessário a fim de humanizar o atendimento, ampliar a oferta à demanda espontânea e evitar a procura pelas emergências dos hospitais ${ }^{19}$.

Diversos estudos realizados no Brasil mostraram que o componente acesso atingiu os escores mais baixos. Em São Luís do Maranhão, os itens A1 (serviços de saúde abertos sábados e domingos) e A2 (serviço de saúde aberto, pelo menos em alguns dias da semana até as 20 horas) apresentaram mais de $80 \%$ de respostas negativas ("com certeza, não") ${ }^{20}$. Estudo realizado em Chapecó envolvendo médicos e enfermeiros como respondentes alcançou escore de 3,6 no atributo acessibilidade ${ }^{21}$. Investigação realizada em Curitiba apresentou resultado similar, em que o escore médio do atributo acessibilidade foi 4,922 . Contudo, resultados encontrados em estudo desenvolvido em Porto Alegre mostraram o atributo acesso com forte extensão à APS. A ESF atingiu escore de 8,6, enquanto as UBS com modelo tradicional alcançaram 7,6, sendo as diferenças estatisticamente significativas ${ }^{23}$.

Ao se considerar a perspectiva dos usuários, estudo desenvolvido em São Paulo apontou que $o$ acesso aos serviços de APS foi dificultado, impactando negativamente a avaliação, em especial no que dizia respeito à resolução de queixas agudas ${ }^{24}$.

Deste modo, estudo realizado por D'Avila et al. ${ }^{25}$ sugeriu a necessidade de atualização do instrumento PCATool no item acessibilidade, uma vez que a comunicação médico-paciente ganhou novas formas nos últimos anos, tais como: e-mails, aplicativos de celulares, software de transmissão de vídeos, formas não contempladas no instrumento original e que poderiam facilitar o acesso dos usuários.

Ao se analisar o atributo coordenação/integração do cuidado, evidenciou-se o baixo grau da APS apresentado pelo componente em ambos os modelos de atenção. Ao se apreciar a composição dos itens utilizados na formação deste atributo, foi possível constatar a marcante deficiência do sistema de referência e contrarreferência no município de Sapucaia do Sul. A totalidade dos profissionais que participaram da pesquisa respondeu que não receberam de forma sistemática informações importantes dos especialistas aos quais tinham encaminhado seus pacientes. É imprescindível para a gestão municipal transpor o desafio de informatizar e integrar a rede de saúde municipal, bem como conscientizar os profissionais em todos os níveis do sistema quanto ao preenchimento adequado dos documentos utilizados como referência e contrarreferência, uma vez que as dificuldades de comunicação entre os diferentes pontos da rede geram encaminhamentos desnecessários, custos operacionais que poderiam ser evitados e que causam grande impacto na vida da população. Deve-se destacar que a plena sintonia entre a referência e contrarreferência é questão relevante e imprescindível para a coordenação dos cuidados ${ }^{17}$.

No presente estudo, os intervalos de confiança dos atributos: integralidade - serviços disponíveis e orientação comunitária nas ESF apresentaram valores superiores aos encontrados nas UBS com modelo tradicional.

$\mathrm{Na}$ avaliação de integralidade - serviços disponíveis, destacou-se a forte extensão apresentada pelas equipes de ESF, enquanto, no modelo tradicional, observou-se escore limítrofe. Para essa avaliação, foram realizadas 22 perguntas aos profissionais de saúde, contudo vale ressaltar que, em relação aos procedimentos de cirurgia ambulatorial, houve um número expressivamente pequeno de respostas positivas que podem ser atribuídas à falta de material 
disponível em todas as unidades ou de treinamento para a realização dos procedimentos específicos. Deve-se ressaltar que a disponibilização dos insumos necessários para a realização de tais procedimentos implica investimento pouco expressivo e pode contribuir para o aperfeiçoamento do atributo.

O atributo orientação comunitária referiu-se aos conhecimentos dos profissionais em relação às necessidades da comunidade na qual a unidade de saúde estava inserida. O melhor desempenho apresentado pelas equipes de ESF foi relacionado principalmente à realização de visitas domiciliares e ao estímulo do controle social através da organização de conselhos locais.

Apenas 2 profissionais que atuavam em UBS tradicional afirmaram realizar visitas domiciliares em sua prática assistencial. Estudo desenvolvido por Van Stralen ${ }^{26}$ corroborou o achado e mostrou que a orientação comunitária foi mais bem desempenhada pelas equipes de ESF, atribuindo esse melhor resultado à presença dos agentes comunitários de saúde nas equipes de ESF.

Os escores observados para o atributo longitudinalidade atingiram valores classificados entre os que apresentaram forte grau de extensão à APS, embora tenham sido encontrados itens no componente que apresentaram baixo valor atribuído nas respostas. A correção dessas medidas aparentemente não implicaria gastos financeiros e aperfeiçoaria a qualidade do acompanhamento dos indivíduos pelas unidades. Sabe-se que garantir o atendimento longitudinal prestado pelos mesmos profissionais e nos mesmos serviços tem proporcionado melhor entendimento e aceitação por parte dos pacientes às orientações médicas, tendendo a diminuir a procura por outros tipos de serviços ${ }^{23}$.

O componente denominado coordenação - sistemas de informação recebeu melhor avaliação, apresentando escores elevados em ambos os modelos de atenção. Esse achado foi também evidenciado em outros estudos que utilizaram o PCATool - Brasil, versão profissionais de saúde ${ }^{22,27,28}$.

O atributo integralidade/serviços prestados se caracteriza por avaliar o processo, ou seja, o desempenho de atividades desenvolvidas pelos profissionais ${ }^{18}$. No presente estudo, o escore obtido foi considerado altamente orientado à APS. Embora o atributo tenha apresentado expressivo valor atribuído, se faz necessário trabalhar questões relativas a ações de prevenção de agravo, como a orientação quanto à guarda segura de armas de fogo. Estudos desenvolvidos por Silva et al. ${ }^{29}$, em Alfenas, MG, e Castro et al..$^{28}$, em Porto Alegre, RS, ratificaram o achado, uma vez que também apresentaram alto grau de extensão à APS no referido atributo, entretanto, estudos de Motizuki ${ }^{30}$; Oliveira ${ }^{31}$ e Rolim ${ }^{32}$, mostraram resultados não favoráveis.

A orientação familiar também atingiu alto valor, demonstrando que os serviços da APS do município de Sapucaia do Sul apresentavam forte orientação para a APS, no que se referiu a este atributo. Para Starfield ${ }^{17}$, a orientação familiar estava relacionada ao reconhecimento de fatores familiares relacionados à gênese e ao tratamento da doença. Desta forma, evidenciou-se que a preocupação com a dinâmica e a organização das famílias estavam incorporadas às práticas dos profissionais da rede da APS do município, denotando a compreensão dentro do contexto social na qual a equipe estava inserida. Estudo de Chomatas ${ }^{22}$ mostrou alto desempenho para APS no atributo orientação familiar. Em contrapartida, estudos de Elias et al.8; Ibãnez', Van Stralen ${ }^{26}$ e Turci; Lima-Costa; Macincko ${ }^{33}$ mostraram resultados não favoráveis ao atributo.

Entre as limitações do presente estudo, pode-se ressaltar que este foi conduzido a partir de respostas dos profissionais enfermeiros, coordenadores das unidades, o que possivelmente estimou positivamente os resultados alcançados. Alguns estudos compararam a avaliação dos usuários com a dos profissionais de saúde. Os resultados mostraram a avaliação dos usuários menos positiva para quase todos os atributos da APS pesquisados ${ }^{26,34,35}$.

Apesar das limitações, o presente estudo identificou ações que precisam ser implantadas para a qualificação da APS no município de Sapucaia do Sul. Ainda que não tenha apontado uma franca supremacia de um modelo sobre o outro no município, deve-se destacar que o instrumento foi adequado para demonstrar a necessidade de facilitar o acesso mediante ampliação dos horários de atendimento, de introduzir novas formas de comunicação com os usuários, de aprimorar e regular os sistemas de referência e contrarreferência, além de elencar ações pouco executadas que certamente contribuem para a efetividade dos cuidados. 


\section{REFERÊNCIAS}

1. Paim J, Travassos C, Almeida C, Bahia L, Macinko J. The Brazilian health system: history, advances, and challenges. Lancet. 2011;377(9779):1778-97. http://dx.doi.org/10.1016/S0140-6736(11)60054-8. PMid:21561655.

2. Santos NR. Desenvolvimento do SUS, rumos estratégicos estratégias para visualização dos rumos. Cien Saude Colet. 2007;12(2):429-35. http://dx.doi.org/10.1590/S1413-81232007000200019. PMid:17680098.

3. Barreto ML, Rassela D, Machado DB, Aquino R, Lima D, Garcia LP, et al. Monitoring and evaluating progress towards universal health coverage in Brazil. PLoS Med. 2014;11(9):1-3. http://dx.doi.org/10.1371/journal. pmed.1001692. PMid:25243676.

4. Macinko J, Lima-Costa MF. Horizontal equity in health care utilization in Brazil, 1998-2008. Int J Equity Health. 2012;11(33):33. http://dx.doi.org/10.1186/1475-9276-11-33. PMid:22720869.

5. Elias PE, Ferreira CW, Alver MCG, Cohn A, Kishima V, Escrivão A Jr, et al. Atenção básica em saúde: comparação entre PSF e UBS por estrato de exclusão social no município de São Paulo. Cien Saude Colet. 2006;11(3):633-41. http://dx.doi.org/10.1590/S1413-81232006000300012.

6. Anversa ETR, Dal PizzolTS, Bastos GAN, Nunes LN. Qualidade do processo da assistência pré-natal: unidades básicas de saúde e unidades de estratégia saúde da família em município no sul do Brasil. Cad Saude Publica. 2012;28(4):789-800. http://dx.doi.org/10.1590/S0102-311X2012000400018. PMid:22488324.

7. Macinko J, Oliveira VB, Turci MA, Guanais FC, Bonolo PF, Lima-Costa MF. The influence of primary care and hospital supply on ambulatory care-sensitive hospitalizations among adults in Brazil, 1999-2007. Am J Public Health. 2011;101(10):1963-70. http://dx.doi.org/10.2105/AJPH.2010.198887. PMid:21330584.

8. Macinko J, Guanais FC, Souza MFM. Evaluation of the impact of the family health program on infant mortality in Brazil, 1990-2002. J Epidemiol Community Health. 2006;60(1):13-9. http://dx.doi.org/10.1136/ jech.2005.038323. PMid:16361449.

9. Ibañez N, Yazle Rocha JS, Castro PC, Ribeiro MCSA, Forster AC, Novaes MHD, et al. Avaliação do desempenho da atenção básica no Estado de São Paulo. Cien Saude Colet. 2006;11(3):683-703. http://dx.doi.org/10.1590/ S1413-81232006000300016.

10. Fracolli LA, Pereira Gomes MF, Nabão FRZ, Santos MS, Cappellini VK, Almeida ACC. Instrumentos de avaliação da Atenção Primária à Saúde: revisão de literatura e metassíntese. Cien Saude Colet. 2014;19(12):4851-60. http://dx.doi.org/10.1590/1413-812320141912.00572014. PMid:25388193.

11. Castanheira ERL, Nemes MIB, Almeida MAS, Puttini RF, Soares ID, Patrício KP, et al. QualiAB: desenvolvimento e validação de uma metodologia de avaliação de serviços de atenção básica. Saude Soc. 2011;20(4):93547. http://dx.doi.org/10.1590/S0104-12902011000400011.

12. Costa NR. A estratégia de saúde da família, a atenção primária e o desafio das metrópoles brasileiras. Cien Saude Colet. 2016;1(5):1389-98. http://dx.doi.org/10.1590/1413-81232015215.24842015. PMid:27166889.

13. Shi L, Starfield B, Xu J. Validating the adult primary care assessment tool. J Fam Pract. 2001;50:161-75.

14. Brasil. Ministério da Saúde. Secretaria de Atenção à Saúde. Departamento de Atenção Básica. Manual do instrumento de avaliação da atenção primária à saúde: primary care assessment tool pcatool-Brasil. Brasília: Ministério da Saúde; 2010.

15. Donabedian A. The quality of care. how can it be assessed? JAMA. 1988;260(12):1743-8. http://dx.doi. org/10.1001/jama.1988.03410120089033. PMid:3045356.

16. Hauser L, Castro RCL, Vigo A, Trindade TG, Gonçalves MR, Stein AT, et al. Tradução, adaptação, validade e medidas de fidedignidade do Instrumento de Avaliação da Atenção Primária à Saúde (PCATool) no Brasil: versão profissionais de saúde. Rev Bras Med Fam Comunidade. 2013;8(29):244-55. http://dx.doi. org/10.5712/rbmfc8(29)821.

17. Starfield B. Atenção primária: equilíbrio entre necessidades de saúde, serviços e tecnologia. Brasília: UNESCO, Ministério da Saúde; 2002.

18. Harzheim E, Starfield B, Rajmil L, Álvarez-Dardet C, Stein AT. Consistência interna e confiabilidade da versão em português do Instrumento de Avaliação da Atenção Primária (PCATool-Brasil) para serviços de saúde infantil. Cad Saude Publica. 2006;22(8):1649-59. http://dx.doi.org/10.1590/S0102-311X2006000800013. PMid:16832536.

19. Almeida PF, Fausto $M C R$, Giovanella L. Fortalecimento da atenção primária à saúde: estratégia para potencializar a coordenação dos cuidados. Rev Panam Salud Publica. 2011;29(2):84-95. http://dx.doi. org/10.1590/S1020-49892011000200003. PMid:21437365.

20. Reis RS, Coimbra LC, Silva AAM, Santos AM, Britto e Alves MTSS, Lamy ZC, et al. Acesso e utilização dos serviços na Estratégia Saúde da Família na perspectiva dos gestores, profissionais e usuários. Cien Saude Colet. 2013;18(11):3321-31. http://dx.doi.org/10.1590/S1413-81232013001100022. PMid:24196897. 
21. Vitoria AM, Harzhein E, Takeda SP, Hauser L. Avaliação dos atributos da atenção primária à saúde em Chapecó, Brasil. Rev Bras Med Fam Comunidade. 2013;8(29):285-93. http://dx.doi.org/10.5712/ rbmfc8(29)832.

22. Chomatas E, Vigo A, Marty I, Hauser L, Harzheim E. Avaliação da presença e extensão dos atributos da atenção primária em Curitiba. Rev Bras Med Fam Comunidade. 2013;29(29):294-306. http://dx.doi. org/10.5712/rbmfc8(29)828.

23. Cunha $\mathrm{CRH}$, Harzheim $\mathrm{E}$, Duncan BB. Percepção da qualidade da atenção à saúde infantil pelos médicos e enfermeiros: comparação entre o programa saúde da família e o modelo tradicional [dissertação]. Porto Alegre: Universidade Federal do Rio Grande do Sul; 2006.

24. Sala A, Luppi CG, Simões O, Marsiglia RG. Integralidade e Atenção Primária à Saúde: avaliação na perspectiva dos usuários de unidades de saúde do município de São Paulo. Saude Soc. 2011;20(4):948-60. http:// dx.doi.org/10.1590/S0104-12902011000400012.

25. D'avila OP, Silva Pinto LF, Hauser L, Gonçalves MR, Harzheim E. O uso do Primary Care Assessment Tool (PCAT): uma revisão integrativa e proposta de atualização. Cien Saude Colet. 2017;22(3):855-65. http:// dx.doi.org/10.1590/1413-81232017223.03312016. PMid:28300993.

26. Van Stralen CJ, Belisário AS, van Stralen TBS, Lima AMD, Massote AW, Oliveira CL. Percepção dos usuários e profissionais de saúde sobre atenção básica: comparação entre unidades com e sem saúde da família na Região Centro- Oeste do Brasil. Cad Saude Publica. 2008;24(Supl. 1):S148-58. http://dx.doi.org/10.1590/ S0102-311X2008001300019. PMid:18660899.

27. Araujo RL, Mendonça AVM, Sousa MF. Percepção dos usuários e profissionais de saúde no Distrito Federal: os atributos da atenção primária. Saúde Debate. 2015;39(105):387-99. http://dx.doi.org/10.1590/0103110420151050002007.

28. Castro RCL, Knauth DR, Harzheim E, Hauser L, Duncan BB. Quality assessment of primary care by health professionals: a comparison of different types of services. Cad Saude Publica. 2012;28(9):1772-84. http:// dx.doi.org/10.1590/S0102-311X2012000900015. PMid:23033191.

29. Silva AS, Nogueira DA, Paraizo CMS, Fracolli LA. Avaliação da atenção primária à saúde: visão dos profissionais de saúde. Rev Esc Enferm. 2014;48:126-132.

30. Motizuki LAC. Análise dos atributos da atenção primária através do primary care assessment tool (pcatoolBrasil). Campo Grande [dissertação]. Campo Grande: Universidade Federal de Mato Grosso do Sul; 2016.

31. Oliveira MMC. Presença e extensão dos atributos da atenção primária à saúde entre os serviços de atenção primária em Porto Alegre: uma análise agregada. [dissertação]: Universidade Federal do Rio Grande do Sul; 2007.

32. Rolim LB. Avaliação dos atributos da atenção primária à saúde de Fortaleza-CE: estudo através do pcatoolbrasil, versão profissionais [dissertação]. Fortaleza: Fundação Oswaldo Cruz, Rede Nordeste de Formação em Saúde da Família (RENASF); 2016.

33. Turci MA, Lima Costa MF, Macinko J. Influência de fatores estruturais e organizacionais no desempenho da atenção primária à saúde em Belo Horizonte, Minas Gerais, Brasil, na avaliação de gestores e enfermeiros. Cad Saude Publica. 2015;31(9):1941-52. http://dx.doi.org/10.1590/0102-311X00132114. PMid:26578018.

34. Carneiro MSM, Melo DMS, Gomes JM, Pinto FJM, Silva MGC. Avaliação do atributo coordenação da Atenção Primária à Saúde: aplicação do PCATool a profissionais e usuários. Saúde Debate. 2014;38:279-295.

35. Silva SA, Baitelo TC, Fracolli LA. Avaliação da Atenção Primária à Saúde: a visão de usuários e profissionais sobre a Estratégia de Saúde da Família. Rev. Latino-Am. Enfermagem. 2015;23(5):979-987. 